\title{
AN 11-YEAR-OLD GIRL PRESENTING WITH CHRONIC KNEE PAIN: A CASE REPORT WITH DIAGNOSTIC DILEMMA
}

\author{
M. Kamal MAJ', Abdul Halim AR ${ }^{1}$, Syed A. Faisal ${ }^{1}$, Johan Ahmad ${ }^{1}$, Srijit Das ${ }^{2}$ \\ University Kebangsaan Malaysia Medical Centre, Faculty of Medicine, Kuala Lumpur, Malaysia: Department of \\ Orthopaedic and Traumatology ${ }^{1}$, Department of Anatomy ${ }^{2}$
}

\begin{abstract}
Summary: Discoid meniscus is the commonest anatomical aberration of the knee joint, among rare cases such as bilateral separated lateral meniscus, accessory lateral meniscus, partial deficiency of the lateral meniscus and double-layered lateral meniscus. An 11-year- old girl presented with history of chronic pain in her right knee for the last 6 months. The problem disturbed her involvement in the sport activities at school. Clinical examination revealed a clicking sensation on knee extension with lateral joint line tenderness. Magnetic resonance imaging (MRI) of her right knee showed torn posterior horn of lateral meniscus. Arthroscopy examination revealed a discoid meniscus with absence posterior horn. Posterior horn deficient discoid meniscus is a rare form of a congenital meniscus anomaly. We as clinicians believe that the abnormal shaped meniscus may pose a diagnostic challenge clinically and radiologically. Presentation of this case may be beneficial for orthopaedicians in their daily clinical practice.
\end{abstract}

Key words: Knee; Anatomy; Pain; Discoid meniscus; Horn; Radiology; Orthopedics

\section{Introduction}

A discoid meniscus is an abnormal discoid, thickened shape fibrocartilaginous meniscus of the knee in contrast to the normal semilunar shape. The lateral menisci is frequently involved. It is also described as the congenital anomaly of menisci. The overall incidence of this disorder ranges between $0.4 \%$ to $16.6 \%$ and it frequently affects the East Asian populations such as Japanese and Koreans $(3,7,9)$.

Other anatomical aberrations of menisci are rare and the presence of posterior horn discoid meniscus is one of rarest congenital anomaly involving the meniscus. The present case report is a humble attempt to highlight the clinical importance of such an anomaly to help orthopaedicians in their future clinical approach.

\section{Case Report}

An 11-year-old girl presented to our clinic with the chief complaint of pain in the right knee for the past 6 months. The pain was felt as a result of trauma. She had slipped and fell on her right knee but recovered well. However, the pain recurred intermittently, which was felt on the anterolateral aspect of her right knee. It was worst on ascending or descending the stairs as well as squatting. She denied any feeling of giving away or locking sensation. The pain forced her to avoid any form of exercise altogether.
On examination, she demonstrated a normal gait. The right knee was not swollen and no joint line tenderness was elicited. Range of movement was $0-140^{\circ}$ but a 'clicking' sound was heard and felt during extension of the knee. Clinically, all the ligaments were intact.

Her right knee radiographs showed a hypoplastic lateral femoral condyle with widening of the lateral joint space. MRI findings reported posterior horn tear of lateral meniscus. No report on discoid meniscus was concluded.

Diagnostic arthroscopy was performed through routine anteromedial and anterolateral portal. Patellofemoral joint and medial compartment were found to be normal. However, lateral compartment revealed a thick, disc-shaped anterior horn of lateral meniscus. On further examination, it was surprisingly found that the posterior horn was completely deficient on the posterior aspect. The anterior and posterior cruciate ligaments were intact. Shaving and debridement of the discoid anterior horn was performed. She was discharged the day after her symptoms relieved. After 6 months post surgery, she remained asymptomatic and was able to take part in her school sport activities.

\section{Discussion}

Discoid meniscus is the commonest anatomical aberration of the knee joint, among rare cases such as bilateral separated lateral meniscus, accessory lateral meniscus, par- 
tial deficiency of the lateral meniscus and double-layered lateral meniscus.

The natural history of this anomaly is still unsure. The origin of discoid meniscus itself was at first believed to be failure of resorption at the central part of the meniscus, but later on disputed. Some researchers were unable to identify a discoid-shaped structure at any stage of normal development in their cadaveric study (2). By $8^{\text {th }}$ week of gestation, the characteristic shape of the menisci was formed $(4,6,10)$. Any disruption of the embryological development before this period might be the cause of the anomaly in the lateral meniscus.

If the discoid meniscus had normal attachment to the tibial plateau, usually it would be asymptomatic and such would not require treatment. The meniscus is 'hypermobile' when it lacks posterior attachment to the tibia, and this may lead to 'snapping' or 'popping' of the knee while flexed and locking in extension as illustrated in our patient. Associated tears may lead to pain. MRI scan can help identifying discoid meniscus and associated meniscal tears.

Reports of the Watanabe classification have been widely accepted worldwide to describe discoid meniscus. It is based on the degree of coverage of the tibial plateau, Type I being incomplete coverage and Type II is complete. Type III or otherwise called Wrisberg-type, is when there is presence of abnormal posterior attachment to posterior cruciate ligament with the disc retaining its crescent shape (9).

A thick discoid meniscus, with its diminished vascular blood supply and in some instances, weak capsular attachment, makes it more prone to tears as compared to a normal meniscus. This is manifested as knee pain and is the reason which drives the patient to seek medical treatment. Another symptom is 'popping' when a loud click or snap heard on knee motions because of the translation of femoral condyles over thickened lateral meniscus (9). Extension block is attributed to the morphology of the meniscus. An earlier study reported that thickened anterior portion of dis-

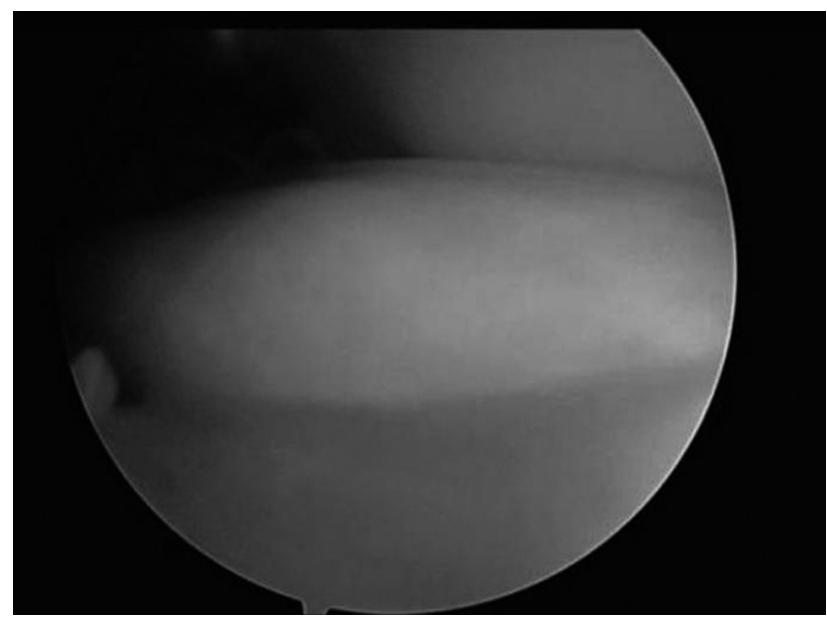

Fig 1: Arthroscopic findings of thickened and disc-shaped lateral meniscus. coid meniscus can be considered as the mechanical factor which limits the knee extension (1).

Radiological investigation was used in diagnosing as well as planning when operative measures were required. A radiograph may show lateral joint space widening, a square lateral femoral condyle and cupping of the lateral tibial plateau $(3,9)$. Silverman's criteria were used to diagnose discoid meniscus on MRI, which was the continuity of anterior and posterior horns in more than 3 contiguous $5 \mathrm{~mm}$ sagittal slices $(7,9)$. This was used on the basis of cadaveric and surgical specimens revealed the mean of normal transverse width is 10.3 and $11.6 \mathrm{~mm}$ respectively with cut off value of $15 \mathrm{~mm}^{3}$.

However, this method may lead to a false negative result when evaluating people with the Wrisberg variant of discoid meniscus since it maintains a narrow crescent shape, as well as when associated with meniscal tears. Other measures include demonstrating free edge of meniscus in intercondylar eminence on coronal slice and counting ratio of meniscus to tibia $>20 \%$ (7).

The aim of the management was to make the child get rid of any pain. Basically, there were two principal goals i.e. immediate relief of symptoms and long-term prevention of osteoarthritis in the affected knee (9). Operative management generally includes meniscal repair, partial or total meniscectomy (3). However, the assumption of total meniscectomy leading to early osteoarthritis has yet to be conclusively proven. In an earlier finding in a 20 -year follow up study, it was observed that 10 of 11 knees showed osteoarthritic changes on x-ray following total meniscectomy (8). Another 17-year follow-up study found 3 out of 9 knees to have mild joint space narrowing in (11). It has been postulated that development of osteoarthritis, after complete meniscectomy may not be as common in children because of the axial alignment of the extensor mechanism, tissue pliability and resulting knee adaptation to the new forces acting on the joint surface (3).

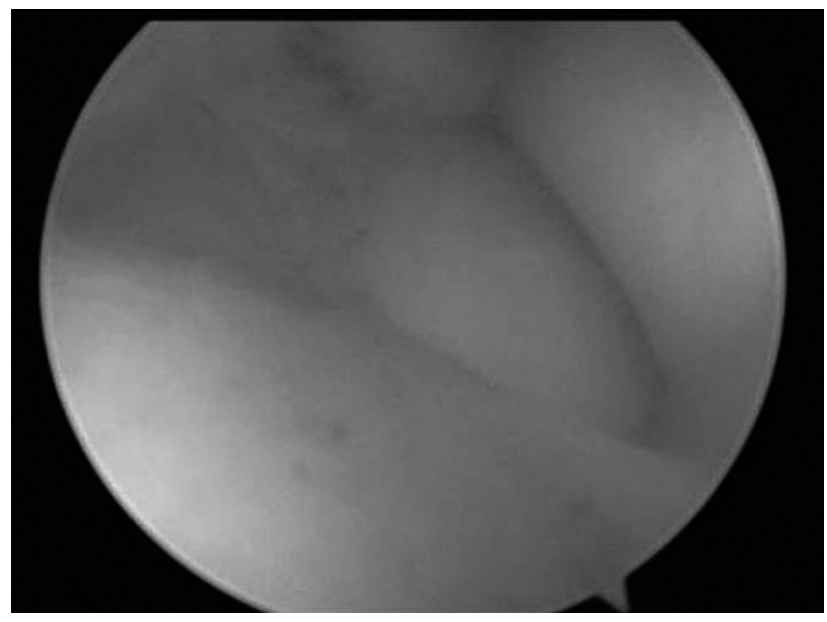

Fig 2: Arthroscopic findings of discoid lateral meniscus with deficient posterior horn. 
An earlier study on 13 children who underwent complete resection, 4 were found to be excellent, 5 had good and another 4 had fair results at an average follow-up of 4 years 7 months (3). Only 2 cases of degenerative change were encountered. Symptomatic cases with recurrent locking or persistent pain can be treated with partial removal of the torn portion, restoring the anatomical configuration of the meniscus or complete resection of the meniscus.

In recent years, cases have been reported regarding other rare aberrations of the lateral meniscus apart from discoid. An earlier study had reported an accessory lateral meniscus where a cartilaginous structure appear from the posterolateral portion of lateral tibial plateau and inserted jointly with the main part of lateral meniscus, which could be the cause of unremitting knee pain in a young lady (4).

Few authors have described a double-layered lateral meniscus. A recent study had found an accessory proximal hemi meniscus overlying the body and posterior horn of the normal lateral meniscus during diagnostic arthroscopy (5). An earlier researcher performed the knee arthroscopy on a 10 year old boy who complained of right knee pain and locking and found that there was an anomalous insertion of anterior horn of medial meniscus while the lateral meniscus was completely separated and duplicated at the midportion (6). They soon performed arthroscopy on the left knee and concluded that this anomaly was actually bilateral.

In our case, the anomaly was due to deficiency of posterior horn while the anterior part was discoid. This was quite similar to an earlier reported case (10). The earlier case reported the anomaly in a 22-year-old male (10). However, the earlier study found that the anterior portion of the meniscus was normal in shape, and there was a partial anterior cruciate ligament tear with a small stump-like tissue next to the anterior cruciate ligament which was considered to be related to the patient's knee pain (10).

\section{Conclusion}

A posterior horn deficient discoid meniscus is a rare form of a congenital meniscus anomaly. We as orthopaedicians believe that the abnormal shaped meniscus may pose a diagnostic challenge clinically and radiologically and prior knowledge of such an anomaly is important.

\section{References}

1. Ahn JH, Shim JS, Hwang $\mathrm{CH}, \mathrm{Oh}$ WH. Discoid lateral meniscus in children: clinical manifestations and morphology. J Pediatr Orthop 2001, 21:812-6.

2. Clark CR, Ogden JA. Development of the menisci of the human knee joint: Morphological changes and their potential role in childhood meniscal injury. J Bone Joint Surg Am 1983,65:538.

3. Davidson D, Letts M, Glasgow R. Discoid meniscus in children: treatment and outcome. Can J Surg 2003, 46:350-8

4. Karahan M, Erol B. Accessory lateral meniscus: a case report. Am. J. Sports Med. 2004; 32: 1973-6.

5. Karataglis D, Dramis A, Learmonth DJA. Double-layered lateral meniscus: A rare anatomical aberration. The Knee 2006,13:415-6.

6. Komatsu N, Yamamoto K, Chosa E. Bilateral congenital separation of the lateral meniscus; A case report. Knee 2008, 15: 330-2

7. Nobuhiko S, Masakazu K, Toshio T, Kunio K. Diagnosis of discoid lateral meniscus of the knee on MR imaging. Rber DA, Friederich NF, Hefti F. Discoid lateral meniscus in children: long-term follow-up, after total meniscectomy. J Bone Joint Surg Am 1998; 80:1579-86

8. Rebello G, Grottkau B, Albright M, Patel D. Discoid Lateral Meniscus: Anatomy and Treatment. Techniques in Knee Surgery 2006, 5:64-69

9. Tetik O, Doral MN, Atay OA, Leblebicioglu G, Türker S. Partial deficiency of the lateral meniscus. Arthroscopy 2003,19:E42.

10. Washington ER $3^{\text {rd }}$, Root L, Liener UC. Discoid lateral meniscus in children long-term follow-up after excision. J Bone Joint Surg Am 1995;77:1357-61.

Received: $18 / 09 / 2009$.

Accepted in revised form: 22/10/2010.

\section{Corresponding author:}

Dr. Muhammad Kamal MAJ, Department of Orthopaedic and Traumatology, Faculty of Medicine, University Kebangsaan Malaysia Medical Centre, Jalan Yaacob Latiff, Bandar Tun Razak, 56000 Cheras, Kuala Lumpur, Malaysia, e-mail: drkortho@gmail.com 